\title{
Evaluación de la productividad académica de las competencias ciudadanas en la enseñanza de la ingeniería por medio del índice Malmquist
}

\author{
Enrique J. Delahoz-Dominguez ${ }^{(1) \star}$, Tomás Fontalvo(2) y Rohemí Zuluaga(1) \\ (1) Universidad Tecnológica de Bolívar, Facultad de Ingenierías, Programa Ingeniería Industrial. Campus de Ternera, \\ Cartagena. Colombia, (correo-e: edelahoz@utb.edu.co; rzuluaga@utb.edu.co). \\ (2) Universidad de Cartagena, Facultad de Ciencias Económicas, Programa Administración Industrial., Piedra de Bolívar \\ Campus, Cartagena. Colombia, (correo-e: tfontalvoh@unicartagena.edu.co).
}

* Autor a quien debe ser dirigida la correspondencia.

Recibido Ene. 16, 2020; Aceptado Mar. 18, 2020; Versión final Abr. 14, 2020, Publicado Oct. 2020

\begin{abstract}
Resumen
Esta investigación analizó la productividad en la formación de competencias ciudadanas en la enseñanza de la ingeniería. Como población se tomó los 92 programas (57.520 estudiantes) que presentaron los exámenes de estado SABER PRO que en Colombia ofrecen carreras de ingeniería industrial, mecánica, civil, sistemas y ambiental para los años 2016, 2017 y 2018. Articulando el análisis de componentes principales y el índice de Malmquist se determinaron los índices de productividad y valores objetivos de mejoramiento. Las variables de entrada son las competencias en razonamiento cuantitativo, lectura crítica, comunicación escrita e Inglés. La variable de respuesta es el resultado de la evaluación en competencia ciudadana. Los resultados muestran que la ingeniería ambiental y civil no fueron productivas para el año 2017. Además, los estudios de ingeniería industrial presentan mayor variabilidad entre periodos. Se concluye que la competencia en inglés es el factor crítico para mejorar la productividad en competencia ciudadana.
\end{abstract}

\section{Evaluation of academic productivity of citizen competencies in the teaching of engineering by using the Malmquist index}

\begin{abstract}
This research analyzed productivity in the formation of citizen skills in engineering education. The population (57,520 students) included 92 Colombian undergraduate engineering degree programs (industrial, mechanical, civil, systems, and environmental) that were examined in the national exam SABER PRO in 2016, 2017, and 2018. Productivity indexes and objective improvement values were determined using the Malmquist index and the principal component analysis. Input variables were competencies in quantitative reasoning, critical reading, written communication, and English. The response variable was the result of the evaluation in citizen competency. The results showed that the environmental and civil engineering programs were not productive in 2017 . Industrial engineering presented the highest variability between periods. It is concluded that English was the critical factor for improving productivity in citizen competency.
\end{abstract}




\section{INTRODUCCIÓN}

La formación de ingenieros en la actualidad está bajo una profunda observación, considerando los reiterados actos de corrupción y malas prácticas en las cuales profesionales de la Ingeniería se han visto involucrados, por ejemplo, en la recopilación de casos desarrollada en (Campello et al., 2018). Para (Leal Filho et al. 2018) el enfoque de transformación no ha sido suficientemente integrado en las instituciones de educación superior, por lo cual es importante conceptualizar cómo la articulación de las competencias de aprendizaje en la enseñanza ingenieril incide en la formación de las habilidades ciudadanas. Ciertamente, los procesos de enseñanza de la Ingeniería han evolucionado, en un principio se trabajaba con un enfoque centrado en el docente, en el cual el profesor era el protagonista del proceso de aprendizaje a través de explicaciones de lo que se debía hacer para aprender (Yamagata 2018). Seguidamente, se generó el paradigma del aprendizaje centrado en el estudiante, en el cual el protagonismo pasa al estudiante y el profesor se convierte en un facilitador de técnicas para lograr la creación de conocimiento desde diferentes aproximaciones (Reigeluth et al. 2015). Así mismo, surgió el enfoque educativo basado en competencias, el cual nació como una tendencia importante desde el siglo XX y aún se considera la base fundamental para la cualificación y desarrollo de profesional, en especial en Latinoamérica, como estrategia de formación humana.

El enfoque de competencias consiste en mejorar la educación por la construcción de capacidades que tengan un efecto positivo en los estudiantes (Fontalvo, De La Hoz 2018), de manera que puedan competir exitosamente en el campo laboral (Lester, Religa 2017). Además, las competencias educativas incitan a desarrollar nuevas metodologías que combinen las tecnologías con las habilidades del trabajador (Lattuca et al., 2017). Por el contrario del modelo centrado en el alumno que enfatiza el aprendizaje individual, el modelo basado en competencias apunta a los resultados (Topping 2016). Sin embargo, el enfoque basado en competencias toma tres principios del enfoque centrado en el alumno: el desarrollo del pensamiento crítico, la resolución de problemas y el aprendizaje significativo. Por lo tanto, en este artículo se concibe el enfoque basado en competencias como un conjunto de conocimientos medibles, habilidades, valores y actitudes relacionadas entre sí que permiten desempeños satisfactorios en situaciones reales de trabajo, según estándares utilizados en un campo de acción (Escobar Valencia 2005). Es así como, antes de ser ingeniero se es una persona, por lo cual las competencias básicas, están presentes en toda la vida del hombre y se cultivan desde la educación primaria. En cambio, las competencias laborales se dividen en generales, específicas y profesionales; las generales son comunes en todos los contextos; las especificas dependen del área de acción y las profesionales, corresponden a los graduados y guardan relación a un desempeño calificado. Finalmente, las competencias ciudadanas son las que debe tener cada persona para actuar de manera constructiva en una sociedad democrática, esta competencia incita al respeto, convivencia y promoción los derechos humanos (Colombo 2018).

El estudio de las competencias ciudadanas en la formación de ingenieros no ha sido profundamente desarrollado, sin embargo, diferentes autores han señalado la importancia de un pensamiento ciudadano global para el éxito profesional de los ingenieros (Cabedo et al., 2018, Gorodetskaya et al., 2015, Kasekende., 2016). Las competencias ciudadanas son definidas por diversos autores como un cumulo de capacidades y comportamientos que buscan de manera integral que todos los individuos puedan ejercer plenamente su ciudadanía a través de valores como respeto, responsabilidad y sentido de pertenencia con el fin de garantizar el cumplimiento de los derechos (Zambrano 2018). Las competencias ciudadanas, se encaminan al avance ecuánime acorde a las habilidades de los educandos, resaltando las capacidades para la toma de decisiones, liderazgo, la resolución de conflictos, la alianza y la cooperación, que deben verse explícitas en toda propuesta pedagógica institucional (Mieles-Barrera y Salgado 2012). Para el caso específico de la enseñanza de la ingeniería, Kagaari y Munene (2007) evidencian la existencia de una relación entre las competencias en ingeniería de los profesores y el desarrollo del comportamiento ciudadano organizacional, evidenciando como a través de la planeación, evaluación y organización de proyectos de Ingeniería se fomentan escrúpulos y valores cívicos entre los estudiantes.

Es así como, Las competencias básicas de un profesional de acuerdo con el Instituto Colombiano para la Evaluación de la Educación (ICFES) son: competencias ciudadanas, comunicación escrita, lectura crítica, inglés y razonamiento cuantitativo. Diversos autores señalan la relación entre competencias y la causalidad entre ellas, por ejemplo, (Samper Ibáñez y Ospino Rihaza, 2018) demuestran como mediante la lectura crítica y la comunicación escrita se puede fortalecer las competencias ciudadanas, por otro lado, (Guilherme 2007) plantea en como la enseñanza del inglés como segunda lengua brinda oportunidades para promover actuaciones de ciudadanos cosmopolitas responsables, sin implicar la perdida de las raíces culturales. $\mathrm{De}$ igual manera, Vanegas (2013) contempla de forma indirecta los criterios que relacionan el proceso de formación ciudadano con las competencias de análisis crítico y concluyen una fuerte relación entre estas dos competencias. Tradicionalmente, cuando en el ámbito educativo se imparten las competencias ciudadanas se busca pasar de la teoría a la práctica, implicando al estudiante en situaciones reales en las cuales pueda contextualizar el aprendizaje ciudadano y medir el impacto de sus decisiones en la comunidad. Por esta razón, 
esta investigación plantea una aproximación objetiva de evaluación de las competencias ciudadanas en la formación de ingenieros. Esta temática ha sido abordada por otras investigaciones, Por ejemplo (Barelli, et al., 2018) han desarrollado el concepto de ciudadanía científica, como resultado de la integración entre ciencia y sociedad, proponiendo la aplicación de conceptos de las ciencias de sistemas complejos en contextos informales de la educación ciudadana. En otra aproximación, el estudio de (Maass., 2019) presenta una investigación interdisciplinaria conectando la enseñanza de la ciencia y la matemática a la educación ciudadana, demostrando que es posible desarrollar habilidades ciudadanas y al mismo tiempo desarrollar las habilidades matemáticas requeridas en el siglo XXI.

Es así como, en la enseñanza de la ingeniería es importante desarrollar competencias ciudadanas y por lo tanto generar esquemas de evaluación que permitan valorar el proceso de aprendizaje en la formación integral de los ingenieros. Consecuentemente, esta investigación propone un análisis de productividad como herramienta de valoración de la apropiación de competencias ciudadanas en el contexto de la enseñanza de la ingeniería en Colombia. De lo anterior se generan los siguientes interrogantes ¿Cuáles son las principales competencias evaluadas en examen Saber Pro que inciden en obtener resultados altos en la Competencia Ciudadana para las universidades estudiadas? ¿En qué medida las competencias evaluadas en los exámenes de estado de los ingenieros inciden en la productividad de las competencias ciudadanas? Considerando lo anterior, el objetivo principal de este estudio es determinar una metodología de evaluación de la formación de Competencias ciudadanas en la enseñanza de la ingeniería, mediante el índice de productividad de Malmquist. Así mismo se establecieron los siguientes objetivos específicos; establecer un conjunto de datos consolidado de la información proveniente del ICFES. Definir las variables para el análisis de productividad. Aplicar los conceptos de productividad para explicar el desarrollo de Competencias ciudadanas en la formación de ingenieros. Identificar las variables críticas para el logro de la productividad asociadas a la Competencia ciudadana en la formación de ingenieros y brindar recomendaciones de mejoramiento.

\section{Aspectos Teóricos}

La primera aproximación a los modelos de medición de la eficiencia con retornos a escala constante fue propuesta por (Charnes, Cooper, Rhodes 1978), conocidos como modelos CCR (Charnes, Cooper, Rhodes). Es así como, a partir de la integración de los modelos DEA como series de tiempo para comparar unidades de decisión (DMU's) se da origen al concepto de indicador de productividad de Malmquist (MPI). De tal forma que, la productividad analizada por estos modelos está definida como el diferencial entre la tecnología (medios) y la eficiencia individual del DMU estudiado. EI MPI, a su vez está compuesto por el indicador de variación de la eficiencia técnica (EC), el cuál mide la variación de la eficiencia de los DMU en entre los periodos estudiados. Y a su vez, también se compone del indicador de variación tecnológica (TC) el cuál mide las aproximaciones o desviaciones de las DMU's a la frontera eficiente. Es así como existen diferentes estudios para medir la productividad de instituciones de educación superior utilizando los modelos de DEA. Por ejemplo, (Visbal-Cadavid et al., 2017) desarrolla un análisis de eficiencia y productividad de universidades en Colombia, considerando variables financieras, académicas y de infraestructura, por su parte (Mehrolhassani et al., 2019) evalúan la eficiencia de las facultades de ciencias en Irán. A su vez, en (Wolszczak-Derlacz 2017) analizaron 500 universidades en 10 países de Europa y Estados Unidos, identificando las universidades de las regiones ricas de Europa como las más eficientes además de mostrar cómo la financiación pública es sinónimo de menor eficiencia. La base del cálculo de los indicadores de productividad son las distancias, es decir, observar que tanto se aleja o se acerca periodo a periodo una DMU a la frontera eficiente. El modelo para el cálculo de la productividad se presenta en las ecuaciones 1, 2 y 3 .

$$
\begin{aligned}
& \operatorname{Mi}\left(x^{t+1}, y^{t+1}, x^{t}, y^{t}\right)=\left(\frac{D_{0}^{t+1}\left(x^{t+1}, y^{t+1}\right)}{D_{0}^{t}\left(x^{t}, y^{t}\right)}\right) *\left[\left(\frac{D_{0}^{t}\left(x^{t+1}, y^{t+1}\right)}{D_{0}^{t+1}\left(x^{t+1}, y^{t+1}\right)}\right)\left(\frac{D_{0}^{t}\left(x^{t}, y^{t}\right)}{D_{0}^{t+1}\left(x^{t}, y^{t}\right)}\right)\right]^{\frac{1}{2}} \\
& e c=\left(\frac{D_{0}^{t+1}\left(x^{t+1}, y^{t+1}\right)}{D_{0}^{t}\left(x^{t}, y^{t}\right)}\right) \\
& t c=\left[\left(\frac{D_{0}^{t}\left(x^{t+1}, y^{t+1}\right)}{D_{0}^{t+1}\left(x^{t+1}, y^{t+1}\right)}\right)\left(\frac{D_{0}^{t}\left(x^{t}, y^{t}\right)}{D_{0}^{t+1}\left(x^{t}, y^{t}\right)}\right)\right]^{\frac{1}{2}}
\end{aligned}
$$

El índice está constituido por dos componentes, el primer componente es la variación de la eficiencia técnica (EC) y el segundo es la variación tecnológica (TC). El componente EC mide los cambios en el nivel de la eficiencia (uso de los recursos académicos e infraestructura) de cada DMU en la transición de un periodo a otro. El segundo componente TC mide los movimientos de la frontera, en otras palabras, los cambios tecnológicos (metodologías y formas de enseñanza) de un periodo a otro. 


\section{METODOLOGÍA}

La presente investigación es de tipo cuantitativa. Se caracteriza formación del ingeniero por medio del método PCA y como eje central se establece un modelo racional que permite evaluar cuantitativamente la productividad de las diferentes competencias para mejorar el desempeño de las competencias ciudadanas de estos ingenieros. Lo anterior utilizando el enfoque de productividad de Malmquist como herramienta de evaluación de la evolución y desempeño de las competencias ciudadanas en diferentes periodos, tomando como criterios de análisis los resultados exámenes universitarios estatales y las académicas del estudiante (Ver Tabla 1). Para lo cual se establece un modelo de Malmquist donde se relacionan estas competencias y su incidencia en las competencias ciudadanas como se observa en la Fig. 1.

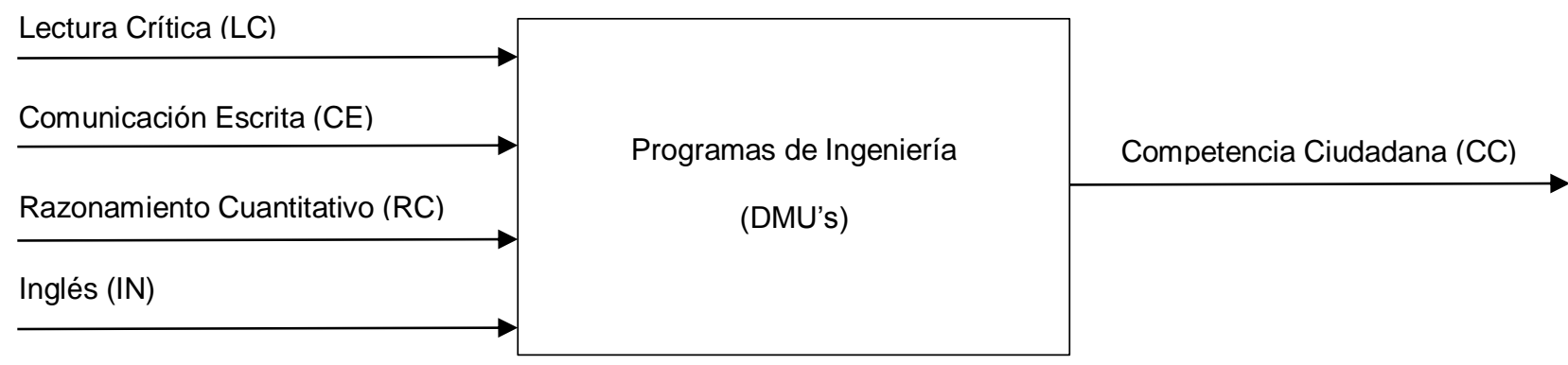

Fig. 1: Relación de variables de entrada y salida del modelo de productividad

Lo anterior, desde de un análisis racional de los autores para conceptualizar las competencias ciudadanas como un factor de productividad, asociadas al desempeño académico en los exámenes universitarios de estado. Así, empíricamente se definen las competencias académicas de Lectura Crítica (LC), Comunicación Escrita (CE), Razonamiento Cuantitativo (RC) e Inglés (ING) como los recursos de formación para adquirir Competencias ciudadanas, definiendo los programas universitarios como las unidades de decisión (DMU). En primer lugar, se plantea un análisis descriptivo apoyado por el análisis de componentes principales (PCA), el cual estudia las variables holísticamente. En segundo lugar, se desarrolla un proceso explicativo e inferencial, soportado por los indicadores del método de productividad de Malmquist, mediante el cual se identifican las relaciones asociativas y causales entre las competencias académicas y la productividad de la competencia ciudadana. Como población para esta investigación se tomó los 92 programas que presentaron los exámenes de estado (SABER PRO) que en Colombia ofrecen carreras de Ingeniería Industrial, Mecánica, Civil, Sistemas y Ambiental para los años 2016, 2017 y 2018. De donde se tomaron los resultados de 57.520 estudiantes que presentaron dichos exámenes. Como fuente de información primaria se tomó la base de datos de los resultados del examen de estado "SABER PRO" en ingeniería en Colombia. Específicamente a los datos publicados por el Instituto Colombiano para el fomento de la educación superior en Colombia (ICFES).

Tabla 1. Descripción de las variables del estudio

\begin{tabular}{|l|l|}
\hline Variable & Descripción \\
\hline $\begin{array}{l}\text { Lectura crítica } \\
\text { (entrada) }\end{array}$ & $\begin{array}{l}\text { Evalúa la capacidad para comprender un texto a nivel local o global y la aproximación } \\
\text { crítica frente a este, valorando las habilidades de un buen lector crítico. }\end{array}$ \\
\hline $\begin{array}{l}\text { Razonamiento } \\
\text { cuantitativo (entrada) }\end{array}$ & $\begin{array}{l}\text { Evalúa la capacidad para comprender y manipular datos cuantitativos en distintas } \\
\text { representaciones, valorando la capacidad de solucionar problemas con datos. }\end{array}$ \\
\hline $\begin{array}{l}\text { Comunicación escrita } \\
\text { (entrada) }\end{array}$ & $\begin{array}{l}\text { Permite determinar en distintos niveles la capacidad que tiene el estudiante de } \\
\text { transmitir de forma escrita sus ideas frente a un tema }\end{array}$ \\
\hline Inglés (entrada) & Evalúa la competencia para comunicarse efectivamente en idioma inglés. \\
\hline $\begin{array}{l}\text { Competencia } \\
\text { ciudadana (salida) }\end{array}$ & $\begin{array}{l}\text { Este módulo evalúa el ejercicio autónomo de la ciudadanía y la coexistencia inclusiva } \\
\text { dentro del marco que propone la constitución colombiana. }\end{array}$ \\
\hline
\end{tabular}

\section{RESULTADOS}

Se desarrolló un PCA para encontrar nuevas variables no correlacionadas que identifiquen relaciones tacitas entre las componentes de conocimiento del estudio, al igual que establecer las variables que conformarán el modelo de productividad de Malmquist. Se realizó una descomposición espectral con el método de los autovalores y auto-vectores. La varianza explicada por las tres primeras componentes es igual a $68.15 \%, 88.14 \%$ y $91.46 \%$ respectivamente. En la primera componente de la Figura 2 (eje horizontal), todas las componentes excepto la de Comunicación Escrita presentan valores superiores a seis puntos, por lo cual a esta se rotulará como el resultado ponderado de las competencias del examen. Así, en la segunda componente principal (Ver Tabla 2) se aprecia como los valores de la Comunicación Escrita para los años 2016, 2017 y 2018 presentan valores de 20.25, 16.45, 19.59 respectivamente, por lo cual esta componente se interpretará como 'Capacidad Comunicativa' en los análisis posteriores de la investigación. 
Tabla 2. Peso de las variables en las componentes principales

\begin{tabular}{|l|l|l|l|l|l|l|l|l|}
\hline Variable & Comp. 1 & Comp. 2 & Variable & Comp. 1 & Comp. 2 & Variable & Comp. 1 & Comp. 2 \\
\hline RC_2016 & 7.62 & 3.61 & RC_2017 & 7.81 & 3.55 & RC_2018 & 7.55 & 3.92 \\
\hline LC_2016 & 8.8 & 0.04 & LC_2017 & 8.15 & 2.94 & LC_2018 & 8.04 & 2.01 \\
\hline CC_2016 & 7.81 & 3.38 & CC_2017 & 6.8 & 5.6 & CC_2018 & 6.78 & 7.01 \\
\hline ING_2016 & 6.96 & 3.73 & ING_2017 & 7.24 & 3.7 & ING_2018 & 6.96 & 4.22 \\
\hline CE_2016 & 2.71 & 20.25 & CE_2017 & 3.8 & 16.45 & CE_2018 & 2.97 & 19.59 \\
\hline
\end{tabular}

EI PCA en esta investigación se realiza con un propósito exploratorio y comparativo, no evaluativo. Por lo cual, en la Tabla 3, en el cuadrante I y IV las variables con dirección de crecimiento positiva son (CE_2016, CE_2017, CE_2018, CC_2016, CC_2017, CC_2018) y (LC_2016, LC_2017, LC_2018, RC_2016, RC_2017, RC_2018, ING_2016, ING_2017, ING_2018) respectivamente. Mientras que, en los cuadrantes II y III no se encuentran variables con dirección de crecimiento positiva.

Tabla 3. Porcentaje de concentración de las observaciones por cuadrante

\begin{tabular}{|l|l|l|l|l|}
\hline Carrera & Cuadrante I & Cuadrante II & Cuadrante III & Cuadrante IV \\
\hline Ing. Ambiental & $3,3 \%$ & $6,7 \%$ & $46,7 \%$ & $43,3 \%$ \\
\hline Ing. Civil & $0,0 \%$ & $0,0 \%$ & $43,3 \%$ & $56,7 \%$ \\
\hline Ing. Mecánica & $0,0 \%$ & $0,0 \%$ & $33,3 \%$ & $66,7 \%$ \\
\hline Ing. Sistemas & $0,0 \%$ & $0,0 \%$ & $63,2 \%$ & $36,8 \%$ \\
\hline
\end{tabular}

En la Figura 2, se observa a los programas de Ingeniería Industrial ubicarse en los cuadrantes I y II, lo cual genera la inquietud las diferencias en contenidos y las enseñanzas de esta carrera con respecto al resto de las carreras de Ingeniería. Sin embargo, al estar las carreras de Ingeniería Industrial posicionalmente relacionadas con las competencias de Comunicación Escrita y Competencia Ciudadana, se puede asociar este hallazgo a los resultados de (Valle y Cabrera 2009), en el cual se identifican las competencias que los estudiantes de ingeniería industrial deberían poseer, destacando las competencias personales e interpersonales como las más relevantes, asociadas a la ética y la capacidad de comunicación. El involucrar las variables asociadas al año de realización de la prueba, aporta trazabilidad al proceso educativo, teniendo en cuenta que los resultados en los procesos de enseñanza toman tiempo en hacerse efectivos.

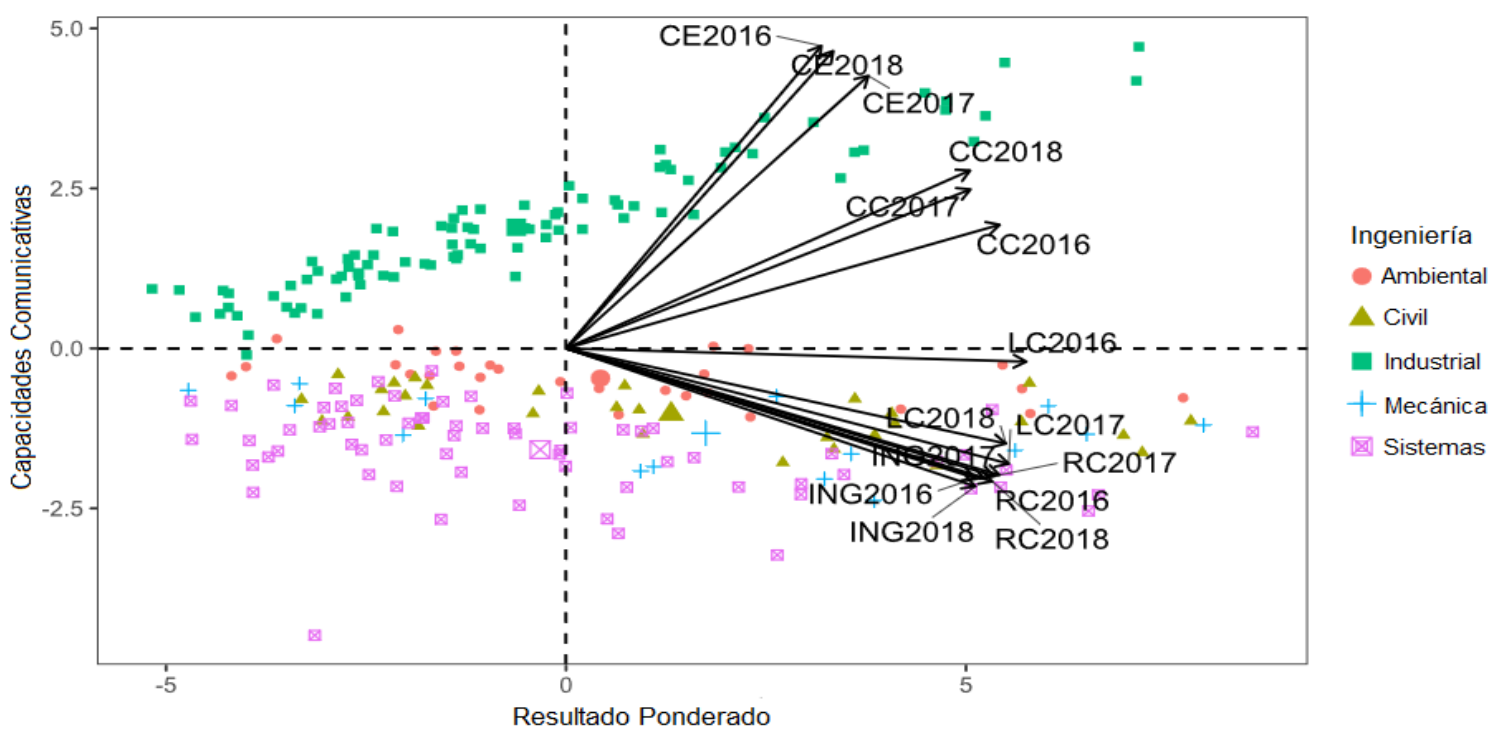

Fig. 2: Representación 2D del PCA

\section{Análisis de productividad}

Los resultados del modelo de productividad se muestran en función de los tres índices del modelo: MI (Malmquist Index), EC (Efficiency Change) y TC (Technical Change). La Tabla 4, Indicadores del modelo de Malmquist, muestra los valores de los índices por periodo, se aprecian comportamientos diferentes en la variación del indicador de productividad del año 2017 al 2018. Para el índice Malmquist (MI) y el índice EC del periodo de 2017, se aprecia como los programas de Ingeniería Ambiental y Civil no fueron productivos. En el índice EC, se evidencia un comportamiento estable entre periodos, excepto para el programa de Ingeniería Industrial (Ver figura 3), el cual tiene la mayor disminución entre periodos. Consecuentemente, Ingeniería Industrial muestra los mayores avances en los indicadores TC Y MI. 
Tabla 4. Indicadores del modelo de Malmquist

\begin{tabular}{|l|l|l|l|l|l|l|}
\hline & \multicolumn{4}{|l|}{2017} & \multicolumn{4}{l|}{2018} \\
\hline Carrera & Ml & EC & TC & Ml & EC & TC \\
\hline Ambiental & 0,998 & 0,998 & 0,999 & 1,016 & 1,022 & 0,994 \\
\hline Civil & 0,980 & 0,990 & 0,989 & 1,028 & 1,031 & 0,997 \\
\hline Mecánica & 1,027 & 1,023 & 1,004 & 0,999 & 1,008 & 0,991 \\
\hline Industrial & 1,021 & 1,044 & 0,979 & 0,997 & 1,005 & 0,992 \\
\hline Sistemas & 1,010 & 1,012 & 0,998 & 1,008 & 1,017 & 0,991 \\
\hline
\end{tabular}

En la Tabla 5, se observa la puntuación promedio que las DMU's deben aumentar para mejorar su productividad. El máximo se refiere al puntaje máximo que alguna universidad del estudio debe alcanzar para lograr ser eficiente. Finalmente, las cantidades de DMU a mejorar se refiere a cuantas universidades (según su carrera) deben mejorar la variable para cumplir los objetivos de eficiencia. Se encuentra que la competencia en el idioma Inglés es la que mayor puntaje objetivo precisa para mejorar la productividad en la enseñanza de las competencias ciudadanas, presentando valores de 8.14, 9.61 y 7.44 para los periodos de 2016, 2017 y 2018 respectivamente.

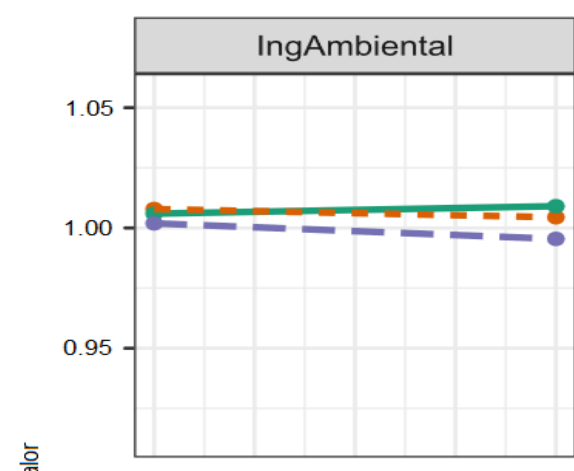

흥

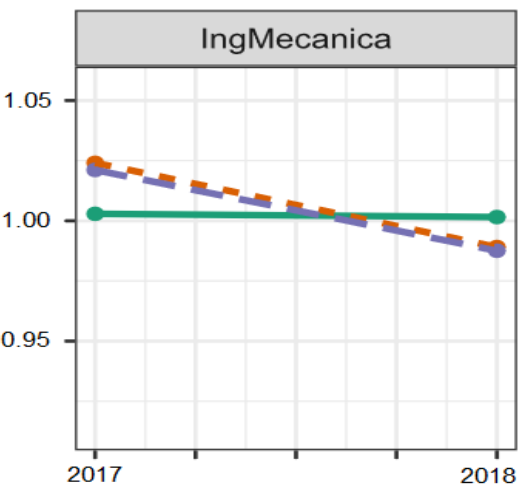

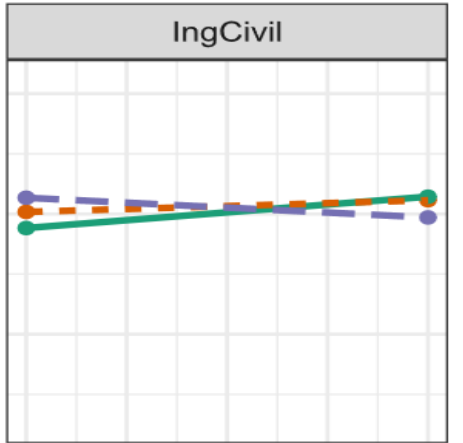
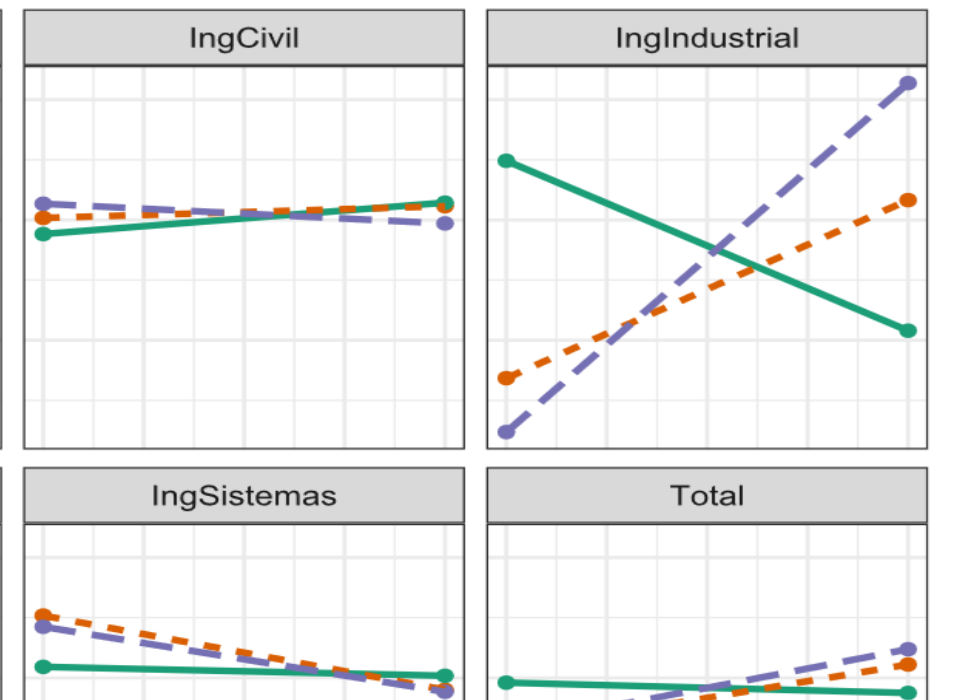

Índice

$\leadsto$ EC

$=\mathrm{MI}$

$\rightarrow$ TC

Fig. 3: Evolución de los índices de productividad 2017-2018

El resultado en la competencia del inglés, es un hallazgo fundamental para comprender el impacto de una segunda lengua en el comportamiento social de un individuo, evidenciando la importancia del concepto de Competencia Ciudadana, como un aspecto global, en los cuáles el contacto con información de diferentes culturas podría consolidar la formación de los ingenieros como ciudadanos globales. Lo anterior, está alineado con las investigaciones (Jaime Osorio et al. 2019) en donde evidenciaron una importante relación entre el proceso de aprendizaje del idioma inglés y el mejoramiento del dialogo y la coexistencia entre los estudiantes. A su vez, (Calle Díaz 2017) realiza una reflexión crítica de la relación entre la enseñanza del inglés y el desarrollo de la educación ciudadana, evidenciando como aprender un idioma permite entender el lenguaje que usamos y el efecto que este tiene en los demás. La metodología desarrollada aporta un valor adicional a los actores involucrados en el proceso de enseñanza de la ingeniería, estudiantes, docentes y directivos. Para los estudiantes esta metodología es útil para seleccionar una universidad de calidad que ofrezca las competencias necesarias para ser un buen profesional, priorizando las competencias de desarrollo ciudadano como factor de decisión. Para los docentes es de gran ayuda para direccionar sus clases al refuerzo de falencias y tener como punto de referencia a otras universidades (Benchmarking). Por último, para los directivos es de gran ayuda para la elaboración de planes de acción que refuercen las falencias de los estudiantes y poder redireccionar el rumbo de sus planes de Carrera. La metodología es un complemento de los trabajos de (Fontalvo-Herrera et al. 2018, Visbal-Cadavid et al. 2019), y puede ser aplicado para cualquier área de educación usando variables académicas. 
Tabla 5. Resumen de los objetivos generados por el análisis de productividad de Malmquist

\begin{tabular}{|c|c|c|c|c|c|}
\hline Ítem & $R C$ & $L C$ & ING & $C E$ & Año \\
\hline Media & 4.98 & 3.88 & 8.14 & 3.13 & \multirow[t]{4}{*}{2016} \\
\hline Desviación & 4.73 & 3.72 & 6.93 & 3.49 & \\
\hline Máximo & 32.53 & 17.12 & 35.90 & 23.83 & \\
\hline Universidades deficientes & 97 & 93 & 149 & 54 & \\
\hline Media & 3.31 & 1.18 & 9.61 & 0.30 & \multirow[t]{4}{*}{2017} \\
\hline Desviación & 3.93 & 2.59 & 7.42 & 1.10 & \\
\hline Máximo & 18.97 & 21.38 & 52.76 & 7.16 & \\
\hline Universidades deficientes & 234 & 234 & 234 & 234 & \\
\hline Media & 7.80 & 0.39 & 7.44 & 1.17 & \multirow[t]{4}{*}{2018} \\
\hline Desviación & 5.79 & 1.77 & 8.88 & 2.71 & \\
\hline Máximo & 22.75 & 16.33 & 68.24 & 18.63 & \\
\hline Universidades deficientes & 234 & 234 & 234 & 234 & \\
\hline
\end{tabular}

\section{CONCLUSIONES}

De acuerdo al trabajo presentado y a los resultados obtenidos, se pueden plantear las siguientes conclusiones principales:

1. La metodología de evaluación y medición de las competencias ciudadanas propuesta para programas académicos aplicado en los programas de ingeniería de Colombia, permite generar una idea de cómo se comportan los datos (PCA), permite evaluar el estado de las DMU's por periodos (DEA - Eficiencia) y la evolución de su desempeño en el tiempo (DEA - Productividad), generando un contexto de información para la toma de decisiones en la gestión universitaria.

2. Es una metodología aplicable a otros contextos de enseñanza universitaria, por lo que es útil para evaluar el proceso de aprendizaje. Esta herramienta permite evaluar las universidades desde dos enfoques: Benchmark (autoevaluación), porque permite analizar si todos los esfuerzos realizados son suficientes para alcanzar la eficiencia, y, por otro lado, Benchmarking (evaluación desde un punto de referencia) porque permite compararte con las otras DMU's y observar que están haciendo para ser eficientes y productivas.

3. Se evidencia cómo la enseñanza del inglés es la competencia evaluada en el examen de estado con mayor oportunidad de mejoramiento en los resultados de productividad de la Competencia Ciudadana, convirtiéndose en el factor crítico para el aumento de la productividad en los programas de ingeniería estudiados, evidenciando la necesidad de promover experiencias de internacionalización dentro de los programas de enseñanza de la ingeniería, para así generar pensamientos globales e interculturales.

\section{NOTACIÓN}

\section{Símbolos}

$\mathrm{M}_{\mathrm{i}}=$ índice de Malmquist

ec $=$ variación de la Eficiencia Técnica

tc $=$ variación Tecnológica

$\mathrm{t}=$ periodo de tiempo

$\mathrm{X}^{\mathrm{t}}=$ entradas del periodo $\mathrm{t}$

$\mathrm{Y}^{\mathrm{t}}=$ salidas del periodo $\mathrm{t}$

$\mathrm{D}_{0}^{\mathrm{t}+1}\left(\mathrm{x}^{\mathrm{t}}, \mathrm{y}^{\mathrm{t}}\right)=$ máximo cambio proporcional (ecs. 1-3)

\section{REFERENCIAS}

Barelli, E., Branchetti, L., Tasquier, G., Albertazzi, L., Levrini, O. Science of Complex Systems and Citizenship Skills: A Pilot Study with Adult Citizens. Eurasia Journal of Mathematics, Science and Technology Education, 14 (4), 1533-1545 (2018)

Cabedo, L., Royo, M., Moliner, L., Guraya, T. University Social Responsibility towards Engineering Undergraduates: The Effect of Methodology on a Service-Learning Experience. Sustainability, 10 (6), 1823. 
Calle Díaz, L. Citizenship Education and the EFL Standards: A Critical Reflection. Profile Issues in Teachers` Professional Development, 19 (1), 155-168 (2018)

Campello, D., Belarmino, K., Thomé, D. Reporting Corruption in Brazil: The Odebrecht Plea Bargain in Jornal Nacional (SSRN Scholarly Paper No. ID 3274967). Rochester, NY: Social Science Research Network (2018)

Charnes, A., Cooper, W. W., Rhodes, E. Measuring the efficiency of decision making units. European Journal of Operational Research, 2 (6), 429-444 (1978)

Colombo, C. Justifications and Citizen Competence in Direct Democracy: A Multilevel Analysis. British Journal of Political Science, 48 (3), 787-806 (2018)

Escobar Valencia, M. LAS COMPETENCIAS LABORALES: ¿La estrategia laboral para la competitividad de las organizaciones? Estudios Gerenciales, 21 (96), 31-55 (2005)

Fontalvo, T. J., De La Hoz, E. J. Diseño e Implementación de un Sistema de Gestión de la Calidad ISO $9001: 2015$ en una Universidad Colombiana. Formación universitaria, 11 (1), 35-44 (2018)

Fontalvo-Herrera, T. J., Delahoz, E. J., Mendoza-Mendoza, A. A., Fontalvo-Herrera, T. J., Delahoz, E. J., MendozaMendoza, A. A. 2018. Aplicación de Minería de Datos para la Clasificación de Programas Universitarios de Ingeniería Industrial Acreditados en Alta Calidad en Colombia. Información tecnológica, 29 (3), 89-96 (2018)

Gorodetskaya, I. M., Shageeva, F. T., Khramov, V. Yu. Development of cross-cultural competence of engineering students as one of the key factors of academic and labor mobility. En 2015 International Conference on Interactive Collaborative Learning (ICL) (2015)

Guilherme, M. English as a Global Language and Education for Cosmopolitan Citizenship. Language and Intercultural Communication, 7 (1), 72-90 (2007)

Jaime Osorio, M. F., Caicedo Muñoz, M. C., Trujillo Bohórquez, I. C. A Radio Program: a Strategy to Develop Students' Speaking and Citizenship Skills. How, 26 (1), 8-33 (2019)

Kagaari, J. R. K., Munene, J. C. Engineering lecturers' competencies and organisational citizenship behaviour (OCB) at Kyambogo University. Journal of European Industrial Training, 31 (9), 706-726 (2007)

Kasekende, F., Munene, J. C., Otengei, S. O., Ntayi, J. M. Linking teacher competences to organizational citizenship behaviour: The role of empowerment. International Journal of Educational Management, 30 (2), $252-270$ (2016)

Lattuca, L. R., Knight, D. B., Ro, H. K., Novoselich, B. J. Supporting the Development of Engineers' Interdisciplinary Competence. Journal of Engineering Education, 106 (1), 71-97.

Leal Filho, W., Raath, S., Lazzarini, B., Vargas, V. R., Souza, L. de, Anholon, R., Quelhas, O. L. G., Haddad, R., Klavins, M., Orlovic, V. L. The role of transformation in learning and education for sustainability. Journal of Cleaner Production, 199, 286-295 (2018)

Lester, S., Religa, J. "Competence" and occupational standards: observations from six European countries. Education + Training, 59 (2), 201-214 (2017)

Maass, K., Doorman, M., Jonker, V., Wijers, M. Promoting active citizenship in mathematics teaching. ZDM, 51 (6), $991-$ 1003 (2019)

Reigeluth, C. M., Aslan, S., Chen, Z., Dutta, P., Huh, Y., Lee, D., Lin, C.-Y., Lu, Y.-H., Min, M., Tan, V., Watson, S. L., Watson, W. R. Personalized Integrated Educational System: Technology Functions for the Learner-Centered Paradigm of Education. Journal of Educational Computing Research, 53 (3), 459-496 (2015)

Samper Ibáñez, J. E., Ospino Rihaza, I. Competencias ciudadanas desde la perspectiva de la Lectura Crítica en la Educación Universitaria. Enfoque Latinoamericano, 1 (2), 63-77 (2018)

Topping, A. Coaching for Communicative Competence: A Student-Focused Approach. Proceedings of the Canadian Engineering Education Association (CEEA) (2016)

Valle, M., Cabrera, P. ¿Qué competencias debe poseer un ingeniero civil industrial? La percepción de los estudiantes. Revista Iberoamericana de Educación, 50 (4), 1-14 (2009)

Vanegas, Y. Competencias ciudadanas y desarrollo profesional en matemáticas. Unversitat de Barcelona (2013)

Visbal-Cadavid, D., Mendoza, A. M., Hoyos, I. Prediction of efficiency in Colombian Higher Education institutions with Data Envelopment Analysis and Neural Networks. Pesquisa Operacional, 39 (2), 261-275 (2019)

Wolszczak-Derlacz, J. An evaluation and explanation of (in)efficiency in higher education institutions in Europe and the U.S. with the application of two-stage semi-parametric DEA. Research Policy, 46 (9), 1595-1605 (2017)

Yamagata, S. Comparing core-image-based basic verb learning in an EFL junior high school: Learner-centered and teacher-centered approaches. Language Teaching Research, 22 (1), 65-93 (2018)

Zambrano, E. L. Prácticas pedagógicas para el desarrollo de competencias ciudadanas. Revista electrónica de investigación educativa, 20 (1), 69-82 (2018) 